\title{
Multi-objective multi-item solid transportation problem with fuzzy inequality constraints
}

\author{
Dipankar Chakraborty ${ }^{1}$, Dipak Kumar Jana ${ }^{2 *}$ and Tapan Kumar Roy ${ }^{3}$
}

\author{
"Correspondence: \\ dipakjana@gmail.com \\ ${ }^{2}$ Department of Applied Science, \\ Haldia Institute of Technology, \\ Purba Midnapur, Haldia, West \\ Bengal 721657, India \\ Full list of author information is \\ available at the end of the article
}

\begin{abstract}
Zimmermann (Int. J. Gen. Syst. 2:209-215, 1976) first introduced the concept of fuzzy inequality in the field of linear programming problem (LPP). But this concept is hardly used in any real life applications of LPP. So, in this paper, a multi-objective multi-item solid transportation problem (MMSTP) with fuzzy inequality constraints is modeled. Representing different preferences of the decision maker for transportation, three different types of models are formulated and analyzed. Fuzzy inequality solid transportation problem is converted to parameter solid transportation problem by an appropriate choice of flexible index, and then the crisp solid transportation problem is solved by the algorithm (Cao in Optimal Models and Methods with Fuzzy Quantities, 2010) for decision values. Fuzzy interactive satisfied method (FISM), global criterion method (GCM) and convex combination method (CCM) are applied to derive optimal compromise solutions for MMSTP by using MatLab and Lingo-11.0. The models are illustrated with numerical examples and some sensitivity analysis is also presented.
\end{abstract}

Keywords: solid transportation problem; fuzzy inequality; global criterion method; fuzzy interactive satisfied method; convex combination method

\section{Introduction}

The solid transportation problem (STP) is a generalization of the traditional transportation problem in which three-dimensional properties (supply, demand, convenience) are taken into account in the objective and constraint set instead of source and destination. The necessity of considering this special type of transportation problem arises when heterogeneous conveyances are available for shipment of products. The STP is used in public distribution systems. In many industrial problems, a homogeneous product is delivered from its origin to a destination by means of different modes of transport called conveyances, such as trucks, cargo flights, goods trains, ships, etc. These conveyances are taken as the third dimension. A solid transportation problem can be converted to a classical transportation problem by considering only a single type of conveyance.

The STP was first introduced by Haley [1] in 1962. In recent years, there have been numerous papers in this area. Some papers only minimize the total transportation cost. For example, Ojha et al. [2], Pramanik et al. [3] considered an STP for an item with fixed charge, vehicle cost and price discounted varying charge. However, in practical programming problems, the decision maker (DM) usually needs to optimize several objectives.

\section{Springer}

(02014 Chakraborty et al.; licensee Springer. This is an Open Access article distributed under the terms of the Creative Commons Attribution License (http://creativecommons.org/licenses/by/2.0), which permits unrestricted use, distribution, and reproduction in any medium, provided the original work is properly cited. 
Thus, the DM cannot obtain the optimal values of all the objectives simultaneously. The growing literature of STP focuses on multiple objective problems, that is, multiple objective solid transportation problems (MOSTPs). For example, Bit et al. [4] used a fuzzy programming approach to solve a MOSTP; Ida et al. [5] presented a neural network method to solve a MOSTP; Gao and Liu [6] developed two-phase fuzzy algorithms to solve multiobjective STP; Tao and Xu [7] developed a class of rough multiple objective programming and its application to a solid transportation problem.

If more than one objective is to be optimized in an STP, then the problem is called multiobjective solid transportation problem (MOSTP). If we consider more than one item, then it is called multi-item solid transportation problem. If we consider more than one item and more than one objective at a time in an STP, then it is called a multi-objective multiitem solid transportation problem (MMSTP). The MMSTP model was given by Kundu et al. [8]. Recently, Pramanik et al. [9] have developed a multi-objective STP in a fuzzy random environment.

Nowadays, in a very often changing market, the business of a single item does not pay much profit to a retailer. For this reason, almost all businessmen in the fields of transportation (Sancak and Salman [10]) do the business of several items. Generally, in all the cases of STP (multi-objective, multi-item and multi-objective multi-item ones), the inequality has been considered as a general inequality. But we can consider this inequality in the fuzzy environment named fuzzy inequality $[11,12]$. Fuzzy inequality means it will essentially satisfy that inequality condition. Flexible index is used (Cao [13]) to convert it into the general inequality, so that it will give you a chance to choose the appropriate decision value. Two algorithms were given by Cao [13] to find the decision value. We have taken one of them to find the decision value. That decision value will give us a more general optimal solution and an optimal value to minimize the objectives.

The following developments are made in the formulation and solution of MMSTP models:

- Various types of examples have been used to illustrate the single-objective fuzzy inequality constraints.

- MMSTP has been solved in a fuzzy inequality constraint environment.

- Three different soft computing techniques FISM, GCM and CCM have been used to make the comparison between optimal solutions in multi-objective problems.

- Two different soft-computing tools (MATLAB and LINGO-11.0) have been used to solve the examples.

The rest of this paper is organized as follows. In Section 2, we recall some preliminary knowledge about fuzzy inequality constraint linear programming. Section 3 provides the notation and assumption used throughout this paper. In Section 4, we formulate multiobjective multi-item LPP with fuzzy inequality constraint and also provides general information about the fuzzy interactive satisfied method, global criterion method and convex combination method. MMSTP with fuzzy inequality constraints and its two special cases are developed in Section 5. In Section 6 we discuss the solution procedure to those models using the fuzzy interactive satisfied method. A numerical example is solved, and results obtained using the mentioned techniques are compared in Section 7. Section 8 summarizes the paper and also discusses the scope of future work. 


\section{Preliminaries about fuzzy inequality constraint linear programming}

Let us consider the fuzzy inequality constraint linear programming (FICP)

$$
\begin{gathered}
\max z=c x \\
\widetilde{L P} \quad \text { s.t. } A x \lesssim b, \\
x \geq 0,
\end{gathered}
$$

its corresponding parameter linear programming is given by (Cao [13])

$$
\begin{gathered}
\max z=c x \\
L P_{\alpha} \quad \text { s.t. } A x \leq b+(1-\alpha) d, \\
x \geq 0,
\end{gathered}
$$

where $\alpha \in[0,1]$ and $d \geq 0$. In the given discussion, we will use $x_{\alpha}$ as an optimal solution, $B_{\alpha}$ denotes an optimal basis and $z_{\alpha}$ denotes an optimal value of linear programming $\left(L P_{\alpha}\right)$.

Definition 2.1 [13] Let $B$ be one of the optimal basis matrices of $\left(L P_{\alpha}\right)$. If an interval $\left[\alpha_{1}, \alpha_{2}\right]$ exists, satisfying that $B$ is an optimal basis matrix of $\left(L P_{\alpha}\right)\left(\forall \alpha \in\left[\alpha_{1}, \alpha_{2}\right]\right)$ while $B$ is not an optimal basis matrix for each $\alpha \notin\left[\alpha_{1}, \alpha_{2}\right]$, we call $\alpha_{1}$ and $\alpha_{2}$ critical values of $\left(L P_{\alpha}\right)$ and $\left[\alpha_{1}, \alpha_{2}\right]$ a characteristic interval.

Theorem $1\left(L P_{\alpha}\right)$ has a finite characteristic interval on the interval $[0,1]$.

Theorem 2 Let $B$ be an optimal basis matrix of $\left(L P_{\alpha}\right)$ on a characteristic interval $\left[\alpha_{1}, \alpha_{2}\right]$. If $\left(B^{-1} b\right)_{i} \neq 0(1 \leq i \leq m)$, then

$$
\begin{aligned}
& \alpha_{1}=\max \left[\frac{\left[B^{-1}(b+d)\right]_{i}}{\left[B^{-1} b\right]_{i}}, 0 \mid\left(B^{-1} d\right)_{i}<0(1 \leq i \leq m)\right], \\
& \alpha_{2}=\min \left[\frac{\left[B^{-1}(b+d)\right]_{i}}{\left[B^{-1} b\right]_{i}}, 0 \mid\left(B^{-1} d\right)_{i}>0(1 \leq i \leq m)\right]
\end{aligned}
$$

are derived, where $\left(B^{-1}(b+d)\right)_{i}$ and $\left(B^{-1} d\right)_{i}$ are the ith components of $B^{-1}(b+d)$ and $B^{-1} d$, respectively.

Property 2.1 Let $B$ be an optimal matrix of $\left(L P_{\alpha}\right)$ on the characteristic interval $\left[\alpha_{i}, \alpha_{j}\right]$. Then $x_{\alpha}=B^{-1}(b+(1-\alpha) d)\left(\alpha_{i} \leq \alpha \leq \alpha_{j}\right)$ is a linear vector function about variable $\alpha$. The optimal value function $z_{\alpha}=C_{B} B^{-1}(b+(1-\alpha) d)$ is a linear function about variable $\alpha$ and decreases with the increase of variable $\alpha$.

Property 2.2 The optimal value of function $z_{\alpha}$ of $\left(L P_{\alpha}\right)$ continues on the interval $[0,1]$.

\subsection{Algorithm for fuzzy inequality constraint linear programming}

Let $z_{1}$ be an optimal value of $\left(L P_{1}\right)$ and $z_{0}$ be an optimal value of $\left(L P_{0}\right), d_{0}=z_{0}-z_{1}>0$. Based on the above conclusion, the algorithm (Cao [13]) of fuzzy linear programming is given as follows. 
Step 1: Let the optimal solutions of $\left(L P_{0}\right)$ and $\left(L P_{1}\right)$ be $x_{0}$ and $x_{1}$, the optimal values of $\left(L P_{0}\right)$ and $\left(L P_{1}\right)$ be $z_{0}$ and $z_{1}$, and the optimal basis matrix of $\left(L P_{0}\right)$ be $B_{0}$.

Step 2: Solve $\left[B_{0}^{-1}(b+(1-\alpha) d)\right]_{i}=0$.

Assume the solution as

$$
\alpha_{1}, \alpha_{2}, \ldots, \alpha_{n-1} \quad\left(0<\alpha_{1}<\alpha_{2}<\cdots<\alpha_{n-1}<1\right) .
$$

Let $\alpha_{0}=1, \alpha_{n}=1, \alpha=\alpha_{1}, k=1$.

Step 3: Solve $\left(L P_{\alpha}\right)$.

Let the optimal value be $Z_{\alpha}$. If $Z_{\alpha} \leq Z_{1}+d_{0} \alpha$, turn to Step 4, otherwise let $k=k+1$, $\alpha=\alpha_{k}$, turn to Step 3 .

Step 4: Solve the optimal decision

$$
\alpha^{*}=\frac{Z_{1} \alpha_{k}-Z_{1} \alpha_{k-1}-Z_{\alpha_{k-1}} \alpha_{k}+Z_{\alpha_{k}} \alpha_{k-1}}{Z_{\alpha_{k}}-Z_{\alpha_{k-1}}-\alpha_{k} d_{0}+\alpha_{k-1} d_{0}} .
$$

Step 5: Solve linear programming $\left(L P_{\alpha^{*}}\right)$, and we can obtain an optimal solution $x_{\alpha^{*}}$ and an optimal value $z_{\alpha^{*}}$.

\section{Example 2.1 Calculate}

$\max 4 x_{1}+3 x_{2}+3 x_{3}$

subject to

$$
\begin{array}{ll} 
& 2 x_{1}+3 x_{2}+2 x_{3} \lesssim 65, \\
& 3 x_{1}+x_{2}+x_{3} \lesssim 61, \\
\widetilde{L P} & x_{2}+x_{3} \lesssim 24 \\
& x_{2} \lesssim 14 \\
& x_{1}+x_{3} \lesssim 10 \\
& x_{1}, x_{2}, x_{3} \geq 0
\end{array}
$$

where $d=(0,0,0,0,7)$.

The corresponding parametric linear programming problem of the above $\widetilde{L P}$ is presented as follows:

$$
\max 4 x_{1}+3 x_{2}+3 x_{3}
$$

subject to

$$
\begin{array}{ll} 
& 2 x_{1}+3 x_{2}+2 x_{3} \leq 65, \\
& 3 x_{1}+x_{2}+x_{3} \leq 61, \\
L P_{\alpha} \quad & x_{2}+x_{3} \leq 24, \\
& x_{2} \leq 14, \\
& x_{1}+x_{3} \leq 10+7(1-\alpha), \\
& 0 \leq \alpha \leq 1, \quad x_{1}, x_{2}, x_{3} \geq 0 .
\end{array}
$$


Now, using algorithm given in Section 2.1, we obtain $Z_{0}=98.833, Z_{1}=82$ and $d_{0}=16.833$ by calculating $\left(L P_{0}\right)$ and $\left(L P_{1}\right)$. The inverse of the optimal matrix in $\left(L P_{0}\right)$ is

$$
B_{0}^{-1}=\left(\begin{array}{ccccc}
1.667 & -0.5 & 0 & 0 & 1.1667 \\
0.333 & 0 & 0 & 0 & -0.6667 \\
-0.5 & 0.5 & 1 & 0 & -0.5 \\
-0.333 & 0 & 0 & 1 & 0.6667 \\
-0.1667 & 0.5 & 0 & 0 & -0.1667
\end{array}\right) \text {. }
$$

Now, calculating the equations $\left[B_{0}^{-1}(65,61,24,14,10-7 \alpha)\right]_{i}=0(i=1,2, \ldots, 5)$, respectively, we obtain $\alpha_{1}=0.0204, \alpha_{2}=0.7857$, assume $\alpha_{0}=0$ and $\alpha_{3}=1$.

Now solving $\left(L P_{\alpha_{1}}\right)$ we get the optimal solution as $x_{1}=16.8571, x_{2}=10.4286$ and $x_{3}=0$ and the optimal value as $Z_{\alpha_{1}}=Z_{0.0204}=98.714$.

Since $Z_{0.0204}=98.714>Z_{1}+0.0204 * 16.833=82.3434$, we must continue to solve the linear programming $\left(L P_{\alpha_{2}}\right)$. By solving $\left(L P_{\alpha_{2}}\right)$, we obtain the optimal solution as $x_{1}=11.5$, $x_{2}=14$ and $x_{3}=0$ and the optimal value as $Z_{\alpha_{2}}=Z_{0.7857}=88$.

Now $Z_{0.7857}=88<Z_{1}+0.7857 * 16.833=95.2257$, so we stop here and calculate optimal decision $\alpha^{*}$.

Now

$$
\begin{aligned}
\alpha^{*} & =\frac{Z_{1} \alpha_{2}-Z_{1} \alpha_{1}-Z_{\alpha_{1}} \alpha_{2}+Z_{\alpha_{2}} \alpha_{1}}{Z_{\alpha_{2}}-Z_{\alpha_{1}}-\alpha_{2} d_{0}+\alpha_{1} d_{0}} \\
& =0.5514 .
\end{aligned}
$$

Now calculating $\left(L P_{0.5514}\right)$, we obtain the optimal solution as $x_{1}=13.1405, x_{2}=12.9063$ and $x_{3}=0$ and the optimal value as $Z_{0.5514}^{*}=91.2811$.

\section{Notations and assumptions}

\subsection{Notations}

In this solid transportation problem, the following notations are used:

(i) $M=$ number of sources of the transportation problem.

(ii) $N=$ number of destinations of the transportation problem.

(iii) $K=$ number of conveyances, i.e., different modes of transportation.

(iv) $a_{i}^{p}=$ amount of product available at $i$ th origin for $p$ th item.

(v) $b_{j}^{p}=$ demand at $j$ th destination of $p$ th item.

(vi) $e_{k}=$ conveyances of the transportation problem.

(vii) $T$ = number of items.

(viii) $x_{i j k}^{p}=$ the amount to be transported from $i$ th origin to $j$ th destination by means of $k$ th conveyance of $p$ th item (decision variables).

(ix) $C_{i j k}^{t p}=$ per unit transportation cost from $i$ th origin to $j$ th destination by $k$ th conveyance of $p$ th item and $t$ th objective.

\subsection{Assumptions}

In this solid transportation problem, the following assumptions are made.

(i) Homogeneous product should be transported from sources to destinations.

(ii) During transportation no items are damaged, i.e., the amount of received items in destination is the same as the one sent from sources. 


\section{Multi-objective multi-item LPP with fuzzy inequality constraint}

Consider the following multi-objective linear programming problems with fuzzy constraint:

$$
\left\{\begin{array}{l}
\min _{x} C_{l}^{(p)} x^{(p)}, \quad l=1,2, \ldots, k \text { and } p=1,2, \ldots, T \\
\text { s.t. }\left\{\begin{array}{l}
A_{i}^{(p)}\left(x^{(p)}\right) \lesssim B_{i}^{(p)}, \quad i=1,2, \ldots, r \text { and } \forall p, \\
x^{(p)} \geq 0 \quad \forall p,
\end{array}\right.
\end{array}\right.
$$

where $x$ is an $n$-dimensional decision variable column vector.

Its corresponding parametric linear programming is given by

$$
\left\{\begin{array}{l}
\min _{x} C_{l}^{(p)} x^{(p)}, \quad l=1,2, \ldots, k \text { and } p=1,2, \ldots, T \\
\text { s.t. }\left\{\begin{array}{l}
A_{i}^{(p)}\left(x^{(p)}\right) \leq B_{i}^{(p)}+V_{i}, \quad i=1,2, \ldots, r \text { and } \forall p, \\
x^{(p)} \geq 0 \quad \forall p,
\end{array}\right.
\end{array}\right.
$$

where $V_{i}(1 \leq i \leq r)$ is a flexible index by an appropriate choice.

Now the solution methodology of a multi-objective decision making problem by converting into a single-objective problem is discussed as follows.

\subsection{Fuzzy interactive satisfied method}

We introduce the interactive fuzzy satisfied method (FISM) proposed by Sakawa [14], Xu and Zhou [15]. We consider the following multi-objective decision-making model:

$$
\left\{\begin{array}{l}
\max \left[G_{i}(x), i=1,2, \ldots, M\right] \\
\text { s.t. } x \in X
\end{array}\right.
$$

The objective function of equation (7) is to maximize $G_{i}(x)$, so for each objective we introduce the fuzzy objective ' $G_{i}(x)$ approximately more than some value', and the membership function is

$$
\mu_{i}\left(G_{i}(x)\right)= \begin{cases}1 & \text { for } G_{i}(x)>G_{i}^{1}, \\ 1-\frac{G_{i}(x)-G_{i}^{0}}{G_{i}^{1}-G_{i}^{0}} & \text { for } G_{i}^{0} \leq G_{i}(x) \leq G_{i}^{1}, \\ 0 & \text { for } G_{i}(x)<G_{i}^{0} .\end{cases}
$$

In equation (8), the membership is 1 and 0 when the values of $G_{i}(x)$ are $G_{i}^{1}$ and $G_{i}^{0}$, respectively,

$$
G_{i}^{1}=\max _{x \in X} G_{i}(x), \quad G_{i}^{0}=\min _{x \in X} G_{i}(x), \quad i=1,2, \ldots, M .
$$

For model $\min _{x \in X} G_{i}(x)$, its optimal solution should be gotten at the boundary of the convex set $X$. If there exists no solution of $\max _{x \in X} G_{i}(x)$ or $\min _{x \in X} G_{i}(x)$, or $G_{i}^{1}=\infty, G_{i}^{0}=-\infty$, the decision maker may set the value of $G_{i}^{1}, G_{i}^{0}$ subjectively. Hence, equation (9) could be transformed into the following form:

$$
\left\{\begin{array}{l}
\min \left[\mu_{1}\left(G_{1}(x)\right), \mu_{2}\left(G_{2}(x)\right), \ldots, \mu_{m}\left(G_{M}(x)\right)\right] \\
\text { s.t. } x \in X
\end{array}\right.
$$


For each objective function $\mu\left(G_{i}(x)\right)$, let the decision maker give the reference value of membership function $\bar{\mu}_{i}$ to reflect the ideal value of membership function. Through solving the minmax problem (10), we obtain an efficient solution of equation (7) as follows:

$$
\left\{\begin{array}{l}
\min \max _{i=1,2, \ldots, M}\left[\bar{\mu}_{i}-\mu_{i}\left(G_{i}(x)\right)\right] \\
\text { s.t. } x \in X .
\end{array}\right.
$$

Equation (11) is equivalent to

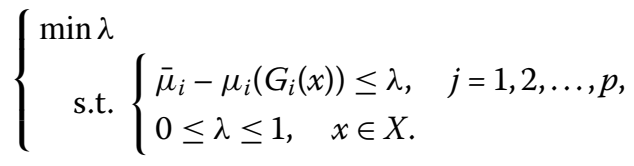

\subsection{Global criteria method}

The global criteria method gives a compromise solution for a multi-objective problem. Actually this method is a way of achieving compromise in minimizing the sum in derivations of the ideal solutions from the respective objective functions. The solution procedure is as follows.

Step-I: Solve the multi-objective problem using each time only one objective $f_{t}$ ( $t=$ $1,2, \ldots, R)$ ignoring all the other objectives.

Step-II: From the results of Step-I, determine the ideal objective vector, say $\left(f_{1}^{\min }, f_{2}^{\min }\right.$, $\left.\ldots, f_{R}^{\min }\right)$ and the corresponding values of $\left(f_{1}^{\max }, f_{2}^{\max }, \ldots, f_{R}^{\max }\right)$.

Step-III: Formulate the following auxiliary problem:

$$
\begin{aligned}
& \min G(x) \\
& \text { subject to } g_{j}(x) \leq 0, \quad j=1,2, \ldots, M, \\
& x \geq 0,
\end{aligned}
$$

where $G(x)=\min \left\{\sum_{t=1}^{R}\left(\frac{f_{t}(x)-f_{t}^{\min }}{f_{t}^{\min }}\right)^{q}\right\}^{\frac{1}{q}}$ or $G(x)=\min \left\{\sum_{t=1}^{R}\left(\frac{f_{t}(x)-f_{t}^{\min }}{f_{t}^{\max }-f_{t}^{\min }}\right)^{q}\right\}^{\frac{1}{q}}$, where $1 \leq q<\infty$. A usual value of $q$ is 2 . This method is then called global criteria method in $L_{2}$ norms.

\subsection{Convex combination method}

We consider the following multi-objective model:

$$
\left\{\begin{array}{c}
\min \left[f_{i}(x), i=1,2, \ldots, M\right] \\
\text { s.t. } g_{j} \geq 0, \quad j=1,2, \ldots, N, \\
x \in X
\end{array}\right.
$$

Then, by the convex combination method, we transfer the above problem into the following form:

$$
\left\{\begin{array}{c}
\min \sum_{i=1}^{M} w_{i} f_{i}(x), \quad \text { where } \sum_{i=1}^{M} w_{i}=1,0<w_{i}<1 \\
\text { s.t. } g_{j} \geq 0, \quad j=1,2, \ldots, N, \\
x \in X .
\end{array}\right.
$$

Corresponding $x$ and $f_{i}(x)$ is solution. 


\section{Formulation of different models of STP}

\subsection{Model-1: multi-objective multi-item STP}

Let $p(p=1,2, \ldots, T)$ items be transported from $M$ origins (or sources) $a_{i}(i=1,2, \ldots, M), N$ destinations (i.e., demands) $b_{j}(j=1,2, \ldots, N)$ and $K$ conveyances $e_{k}(k=1,2, \ldots, K) . K$ conveyances, i.e., different modes of transport, may be trucks, cargo flights, goods trains, ships, etc. Let $a_{i}^{(p)}$ be the product available at $i$ th origin for items $p(p=1,2, \ldots, T), b_{j}^{(p)}$ be the demand at $j$ th destination for items $p(p=1,2, \ldots, T)$, and let $e_{k}$ represent the amount of product which can be carried by $k$ th conveyance. The variable $x_{i j k}^{(p)}$ represents the unknown quantity to be transported from origin $a_{i}^{(p)}$ to destination $b_{j}^{(p)}$ by means of $k$ th conveyance for item $p=1,2, \ldots, T$. Then we propose the mathematical model for the fuzzy inequality constraint. Single-objective and $p(=1,2, \ldots, T)$-item problem is to minimize the total transportation cost as follows:

$$
f_{t}^{(p)}=\sum_{i=1}^{M} \sum_{j=1}^{N} \sum_{k=1}^{K} c_{i j k}^{(t p)} x_{i j k}^{(p)} \quad \forall p \text { and } \forall t .
$$

From the discussion above, we develop mathematical formulations of the objectives as follows:

$$
\min f_{t}=\sum_{p=1}^{T} \sum_{i=1}^{M} \sum_{j=1}^{N} \sum_{k=1}^{K} c_{i j k}^{(t p)} x_{i j k}^{(p)} \quad \forall t .
$$

As mentioned by Haley [1], the constraints are divided into three types: source constraint, destination constraint and conveyance capacity constraint. In the fuzzy environment, the quantity from a source is essentially less than equal to the supply capacity of products for different items, that is,

$$
\sum_{j=1}^{N} \sum_{k=1}^{K} x_{i j k}^{(p)} \lesssim a_{i}^{(p)}, \quad i=1,2,3, \ldots, M \forall p .
$$

In the fuzzy environment, the quantity of product transported to a destination is essentially greater than equal to its demand for different items, that is,

$$
\sum_{i=1}^{M} \sum_{k=1}^{K} x_{i j k}^{(p)} \gtrsim b_{j}^{(p)}, \quad j=1,2,3, \ldots, N \forall p .
$$

In the fuzzy environment, the transportation quantity of conveyance is essentially less than equal to its capacity, that is,

$$
\sum_{p=1}^{T} \sum_{i=1}^{M} \sum_{j=1}^{N} x_{i j k}^{(p)} \lesssim e_{k}, \quad k=1,2,3, \ldots, K .
$$

It is natural to require the nonnegativity of decision variable $x_{i j k}^{(p)}$, that is,

$$
x_{i j k}^{(p)} \geq 0 \quad \forall i, j, k \text { and } \forall p .
$$

It is noted that the decision maker (DM) and the modeling analyst are often different individuals. In the transportation problem, the DM is the manager of transport enterprise, 
while the modeling analyst may be an expert in transportation problems or a researcher in the enterprise. With the complexity of feasible region, the DM may give an appropriately large region so that all the feasible solutions are included in it. Hence, the above MMSTP with fuzzy inequality constraint can be written as

$$
\begin{aligned}
\min f_{t} & =\min \sum_{p=1}^{T} \sum_{i=1}^{M} \sum_{j=1}^{N} \sum_{k=1}^{K} c_{i j k}^{(t p)} x_{i j k}^{(p)}, \quad t=1,2, \ldots, R \\
\text { s.t. } & \sum_{j=1}^{N} \sum_{k=1}^{K} x_{i j k}^{(p)} \lesssim a_{i}^{(p)}, \quad i=1,2,3, \ldots, M \forall p, \\
& \sum_{i=1}^{M} \sum_{k=1}^{K} x_{i j k}^{(p)} \gtrsim b_{j}^{(p)}, \quad j=1,2,3, \ldots, N \forall p, \\
& \sum_{p=1}^{T} \sum_{i=1}^{M} \sum_{j=1}^{N} x_{i j k}^{(p)} \lesssim e_{k}, \quad k=1,2,3, \ldots, K, \\
& x_{i j k}^{(p)} \geq 0 \quad \forall i, j, k,
\end{aligned}
$$

where $\lesssim$ means 'essentially smaller than equal to' and $\gtrsim$ means 'essentially greater than equal to.'

\subsection{Model-2: multi-objective single-item STP}

We consider a multi-objective single-item solid transportation problem with fuzzy inequality constraint. Then the model may be written as

$$
\begin{aligned}
\min f_{t} & =\min \sum_{i=1}^{M} \sum_{j=1}^{N} \sum_{k=1}^{K} c_{i j k}^{t p} x_{i j k} \\
\text { s.t. } & \sum_{j=1}^{N} \sum_{k=1}^{K} x_{i j k} \lesssim a_{i}, \quad i=1,2,3, \ldots, M, \\
& \sum_{i=1}^{M} \sum_{k=1}^{K} x_{i j k} \gtrsim b_{j}, \quad j=1,2,3, \ldots, N, \\
& \sum_{i=1}^{M} \sum_{j=1}^{N} x_{i j k} \lesssim e_{k}, \quad k=1,2,3, \ldots, K, \\
& x_{i j k} \geq 0 \quad \forall i, j, k .
\end{aligned}
$$

\subsection{Model-3: single-objective multi-item STP}

We consider a single-objective multi-item solid transportation problem with fuzzy inequality constraint. Then the model may be written as

$$
\begin{aligned}
\min f & =\min \sum_{p=1}^{T} \sum_{i=1}^{M} \sum_{j=1}^{N} \sum_{k=1}^{K} c_{i j k}^{(p)} x_{i j k}^{(p)} \\
\text { s.t. } & \sum_{j=1}^{N} \sum_{k=1}^{K} x_{i j k}^{(p)} \lesssim a_{i}^{(p)}, \quad i=1,2,3, \ldots, M \forall p,
\end{aligned}
$$




$$
\begin{aligned}
& \sum_{i=1}^{M} \sum_{k=1}^{K} x_{i j k}^{(p)} \gtrsim b_{j}^{(p)}, \quad j=1,2,3, \ldots, N \forall p, \\
& \sum_{p=1}^{T} \sum_{i=1}^{M} \sum_{j=1}^{N} x_{i j k}^{(p)} \lesssim e_{k}, \quad k=1,2,3, \ldots, K, \\
& x_{i j k}^{(p)} \geq 0 \quad \forall i, j, k .
\end{aligned}
$$

\section{Solution of proposed models}

\subsection{Model-1}

Let us consider that $p$ different items are to be transported from $i$ th origin to $j$ th destination by means of $k$ th conveyance. Here we have considered a two-objective function. Let $\max _{x \in X} f_{1}=f_{1}^{U}, \min _{x \in X} f_{1}=f_{1}^{L}, \max _{x \in X} f_{2}=f_{2}^{U}$ and $\min _{x \in X} f_{2}=f_{2}^{L}$. Then the membership functions of $f_{1}$ and $f_{2}$ are given by

$$
\begin{aligned}
& \mu_{1}\left(f_{1}(x)\right)= \begin{cases}0 & \text { for } f_{1}(x)>f_{1}^{U}, \\
\frac{f_{1}^{U}-f_{1}(x)}{f_{1}^{U}-f_{1}^{L}} & \text { for } f_{1}^{L}<f_{1}(x)<f_{1}^{U}, \\
1 & \text { for } f_{1}(x)<f_{1}^{L},\end{cases} \\
& \mu_{2}\left(f_{2}(x)\right)= \begin{cases}0 & \text { for } f_{2}(x)>f_{2}^{U}, \\
\frac{f_{2}^{U}-f_{2}(x)}{f_{2}^{U}-f_{2}^{L}} & \text { for } f_{2}^{L}<f_{2}(x)<f_{2}^{U}, \\
1 & \text { for } f_{2}(x)<f_{2}^{L} .\end{cases}
\end{aligned}
$$

Now, using FISM in Section 4.1, we present the equivalent crisp linear programming of (21) as follows:

$$
\left\{\begin{aligned}
\min _{x} \lambda & \left\{\begin{array}{l}
f_{1} \leq f_{1}^{U}-\left(\mu_{1}-\lambda\right)\left(f_{1}^{U}-f_{1}^{L}\right), \\
f_{2} \leq f_{2}^{U}-\left(\mu_{2}-\lambda\right)\left(f_{2}^{U}-f_{2}^{L}\right), \\
\sum_{j=1}^{N} \sum_{k=1}^{K} x_{i j k}^{(p)} \leq a_{i}^{(p)}+(1-\alpha) d_{i}^{(p)}, \quad i=1,2,3, \ldots, M, \\
\sum_{i=1}^{M} \sum_{k=1}^{K} x_{i j k}^{(p)} \geq b_{j}^{(p)}+(1-\alpha) v_{j}^{(p)}, \quad j=1,2,3, \ldots, N, \\
\sum_{p=1}^{T} \sum_{i=1}^{M} \sum_{j=1}^{N} x_{i j k}^{(p)} \leq e_{k}+(1-\alpha) u_{k}, \quad k=1,2,3, \ldots, K, \\
0 \leq \alpha \leq 1, \\
x_{i j k}^{(p)} \geq 0 \quad \forall i, j, k \text { and } \forall p .
\end{array}\right.
\end{aligned}\right.
$$

\subsection{Model-2}

Let $\max _{x \in X} f_{1}=f_{1}^{U}, \min _{x \in X} f_{1}=f_{1}^{L}, \max _{x \in X} f_{2}=f_{2}^{U}$ and $\min _{x \in X} f_{2}=f_{2}^{L}$. Then the membership functions of $f_{1}$ and $f_{2}$ are given by

$$
\begin{aligned}
& \mu_{1}\left(f_{1}(x)\right)= \begin{cases}0 & \text { for } f_{1}(x)>f_{1}^{U}, \\
\frac{f_{1}^{U}-f_{1}(x)}{f_{1}^{U}-f_{1}^{L}} & \text { for } f_{1}^{L}<f_{1}(x)<f_{1}^{U}, \\
1 & \text { for } f_{1}(x)<f_{1}^{L},\end{cases} \\
& \mu_{2}\left(f_{2}(x)\right)= \begin{cases}0 & \text { for } f_{2}(x)>f_{2}^{U}, \\
\frac{f_{2}^{U}-f_{2}(x)}{f_{2}^{U}-f_{2}^{L}} & \text { for } f_{2}^{L}<f_{2}(x)<f_{2}^{U}, \\
1 & \text { for } f_{2}(x)<f_{2}^{L} .\end{cases}
\end{aligned}
$$


Now, using FISM in Section 4.1, we present the equivalent crisp linear programming of (22) as follows:

$$
\left\{\begin{aligned}
\min _{x} \lambda & \left\{\begin{array}{l}
f_{1} \leq f_{1}^{U}-\left(\mu_{1}-\lambda\right)\left(f_{1}^{U}-f_{1}^{L}\right), \\
f_{2} \leq f_{2}^{U}-\left(\mu_{2}-\lambda\right)\left(f_{2}^{U}-f_{2}^{L}\right), \\
\sum_{j=1}^{N} \sum_{k=1}^{K} x_{i j k} \leq a_{i}+(1-\alpha) d_{i}, \quad i=1,2,3, \ldots, M, \\
\sum_{i=1}^{M} \sum_{k=1}^{K} x_{i j k} \geq b_{j}+(1-\alpha) v_{j}, \quad j=1,2,3, \ldots, N \\
\sum_{i=1}^{M} \sum_{j=1}^{N} x_{i j k}^{(p)} \leq e_{k}+(1-\alpha) u_{k}, \quad k=1,2,3, \ldots, K, \\
0 \leq \alpha \leq 1, \\
x_{i j k}^{(p)} \geq 0 \quad \forall i, j, k .
\end{array}\right.
\end{aligned}\right.
$$

\subsection{Model-3}

Now corresponding parametric linear programming of equation (23) is presented as follows:

$$
\begin{aligned}
\min & \sum_{p=1}^{T} \sum_{i=1}^{M} \sum_{j=1}^{N} \sum_{k=1}^{K} c_{i j k}^{(p)} x_{i j k}^{(p)} \\
\text { s.t. } & \sum_{j=1}^{N} \sum_{k=1}^{K} x_{i j k}^{(p)} \leq a_{i}^{(p)}+(1-\alpha) d_{i}^{(p)}, \quad i=1,2,3, \ldots, M \text { and } \forall p, \\
& \sum_{i=1}^{M} \sum_{k=1}^{K} x_{i j k}^{(p)} \geq b_{j}^{(p)}+(1-\alpha) v_{j}^{(p)}, \quad j=1,2,3, \ldots, N \text { and } \forall p, \\
& \sum_{p=1}^{T} \sum_{i=1}^{M} \sum_{j=1}^{N} x_{i j k}^{(p)} \leq e_{k}+(1-\alpha) u_{k}, \quad k=1,2,3, \ldots, K, \\
& 0 \leq \alpha \leq 1, \quad x_{i j k} \geq 0 \quad \forall i, j, k,
\end{aligned}
$$

where $d_{i}^{(p)}$ for $i=1,2,3, \ldots, M, v_{j}^{p}$ for $j=1,2,3, \ldots, N$ and $u_{k}$ for $k=1,2,3, \ldots, K$ are flexible index values $\forall p$.

\section{Numerical experiment}

\subsection{Input data for Model-1 and Model-3}

Let us consider a multi-objective multi-item solid transportation problem with two types of items (i.e., $T=2$ ), three origins (i.e., $M=3$ ), two destinations (i.e., $N=2$ ) and two types of conveyances (i.e., $K=2)$. The parameters are given as follows.

Transportation cost for 1st objective and 1st item $\left[c_{i j k}^{(11)}\right]$

$$
\left[\begin{array}{lll}
c_{111}^{(11)} & c_{112}^{(11)} & c_{121}^{(11)} \\
c_{122}^{(11)} & c_{131}^{(11)} & c_{132}^{(11)} \\
c_{211}^{(11)} & c_{212}^{(11)} & c_{221}^{(11)} \\
c_{222}^{(11)} & c_{231}^{(11)} & c_{232}^{(11)}
\end{array}\right]=\left[\begin{array}{lll}
11 & 10 & 11 \\
13 & 11 & 10 \\
10 & 11 & 13 \\
14 & 10 & 12
\end{array}\right] .
$$


Transportation cost for 1 st objective and 2 nd item $\left[c_{i j k}^{(12)}\right]$

$$
\left[\begin{array}{ccc}
c_{111}^{(12)} & c_{112}^{(12)} & c_{121}^{(12)} \\
c_{122}^{(12)} & c_{131}^{(12)} & c_{132}^{(12)} \\
c_{211}^{(12)} & c_{212}^{(12)} & c_{221}^{(12)} \\
c_{222}^{(12)} & c_{231}^{(12)} & c_{232}^{(12)}
\end{array}\right]=\left[\begin{array}{lll}
18 & 11 & 12 \\
12 & 13 & 15 \\
12 & 11 & 13 \\
14 & 15 & 12
\end{array}\right]
$$

Transportation cost for 2 nd objective and 1 st item $\left[c_{i j k}^{(11)}\right]$

$$
\left[\begin{array}{lll}
c_{111}^{(21)} & c_{112}^{(21)} & c_{121}^{(21)} \\
c_{122}^{(21)} & c_{131}^{(21)} & c_{132}^{(21)} \\
c_{211}^{(21)} & c_{212}^{(21)} & c_{221}^{(21)} \\
c_{222}^{(21)} & c_{231}^{(21)} & c_{232}^{(21)}
\end{array}\right]=\left[\begin{array}{lll}
16 & 52 & 32 \\
21 & 14 & 26 \\
25 & 35 & 25 \\
11 & 24 & 13
\end{array}\right] .
$$

Transportation cost for 2 nd objective and 2 nd item $\left[c_{i j k}^{(12)}\right]$

$$
\left[\begin{array}{lll}
c_{111}^{(22)} & c_{112}^{(22)} & c_{121}^{(22)} \\
c_{122}^{(22)} & c_{131}^{(22)} & c_{132}^{(22)} \\
c_{211}^{(22)} & c_{212}^{(22)} & c_{221}^{(22)} \\
c_{222}^{(22)} & c_{231}^{(22)} & c_{232}^{(22)}
\end{array}\right]=\left[\begin{array}{ccc}
22 & 19 & 15 \\
18 & 19 & 13 \\
22 & 14 & 12 \\
11 & 8 & 15
\end{array}\right]
$$

Amount of items available at origin $\left[a_{i}^{p}\right]$

$$
\left[\begin{array}{ll}
a_{1}^{(1)} & a_{2}^{(1)} \\
a_{1}^{(2)} & a_{2}^{(2)}
\end{array}\right]=\left[\begin{array}{ll}
35 & 30 \\
28 & 30
\end{array}\right] .
$$

The demand amount of items at destination $\left[b_{j}^{(p)}\right]$

$$
\left[\begin{array}{lll}
b_{1}^{(1)} & b_{2}^{(1)} & b_{3}^{(1)} \\
b_{1}^{(2)} & b_{2}^{(2)} & b_{3}^{(1)}
\end{array}\right]=\left[\begin{array}{lll}
9 & 7 & 8 \\
7 & 7 & 6
\end{array}\right] \text {. }
$$

Amount of items transported by conveyances $\left[c_{k}\right]$

$$
\left[\begin{array}{ll}
e_{1} & e_{2}
\end{array}\right]=\left[\begin{array}{ll}
25 & 26
\end{array}\right] \text {. }
$$

Optimum result for Model-1

With the above input data, $f_{1}$ and $f_{2}$ are calculated using GRG, and we get

$$
f_{1}^{U}=691.5, \quad f_{1}^{L}=602.5, \quad f_{2}^{U}=1,106, \quad f_{2}^{L}=670 .
$$

So, we can get the membership functions of $f_{1}$ and $f_{2}$ (Figure 1 ) as follows:

$$
\begin{aligned}
& \mu_{1}\left(f_{1}(x)\right)= \begin{cases}0 & \text { for } f_{1}(x)>691.5 \\
\frac{691.5-f_{1}(x)}{691.5-602.5} & \text { for } 602.5<f_{1}(x)<691.5 \\
1 & \text { for } f_{1}(x)<602.5\end{cases} \\
& \mu_{2}\left(f_{2}(x)\right)= \begin{cases}0 & \text { for } f_{2}(x)>1,106, \\
\frac{1,106-f_{2}(x)}{1,106-670} & \text { for } 1,106<f_{2}(x)<670, \\
1 & \text { for } f_{2}(x)<670 .\end{cases}
\end{aligned}
$$



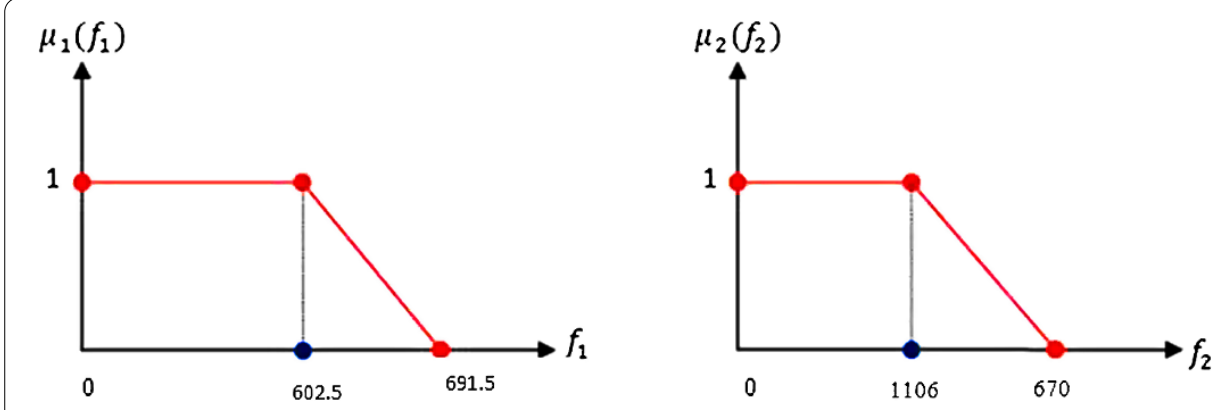

Figure 1 Membership functions of $f_{1}$ and $f_{2}$.

Table 1 Employ the interactive fuzzy satisfied method based on the fuzzy inequality constraint of Model-1

\begin{tabular}{llllllll}
\hline $\boldsymbol{\mu}_{\mathbf{1}}$ & $\boldsymbol{\mu}_{\mathbf{2}}$ & $\boldsymbol{f}_{\mathbf{1}}$ & $\boldsymbol{f}_{\mathbf{2}}$ & $\boldsymbol{\mu}_{\mathbf{1}}\left(\boldsymbol{f}_{\mathbf{1}}\right)$ & $\boldsymbol{\mu}_{\mathbf{2}}\left(\boldsymbol{f}_{\mathbf{2}}\right)$ & Values of decision variables & $\boldsymbol{\lambda}$ \\
\hline 1 & 1 & 634.3 & 826.09 & 0.641 & 0.641 & $\begin{array}{l}x_{111}^{1}=9, x_{121}^{1}=2.04, x_{131}^{1}=8, x_{222}^{1}=4.95, \\
x_{121}^{2}=9.45, x_{122}^{2}=1.04, x_{212}^{2}=10.5, x_{232}^{2}=9.50\end{array}$ & 0.35 \\
1 & 0.9 & 630.98 & 853.13 & 0.679 & 0.579 & $\begin{array}{l}x_{111}^{1}=9, x_{121}^{1}=3.17, x_{131}^{1}=8, x_{222}^{1}=3.82, \\
x_{121}^{2}=8.32, x_{122}^{2}=2.17, x_{212}^{2}=10.5, x_{232}^{2}=9.5\end{array}$ & 0.32 \\
0.9 & 1 & 637.76 & 799.15 & 0.603 & 0.703 & $\begin{array}{l}x_{111}^{1}=9, x_{121}^{1}=0.91, x_{131}^{1}=8, x_{222}^{1}=6.08, \\
x_{121}^{2}=10.5, x_{212}^{2}=10.5, x_{232}^{2}=9.5\end{array}$ & 0.296 \\
& & & & & &
\end{tabular}

Then we compute the following model to get the interactive satisfied solution:

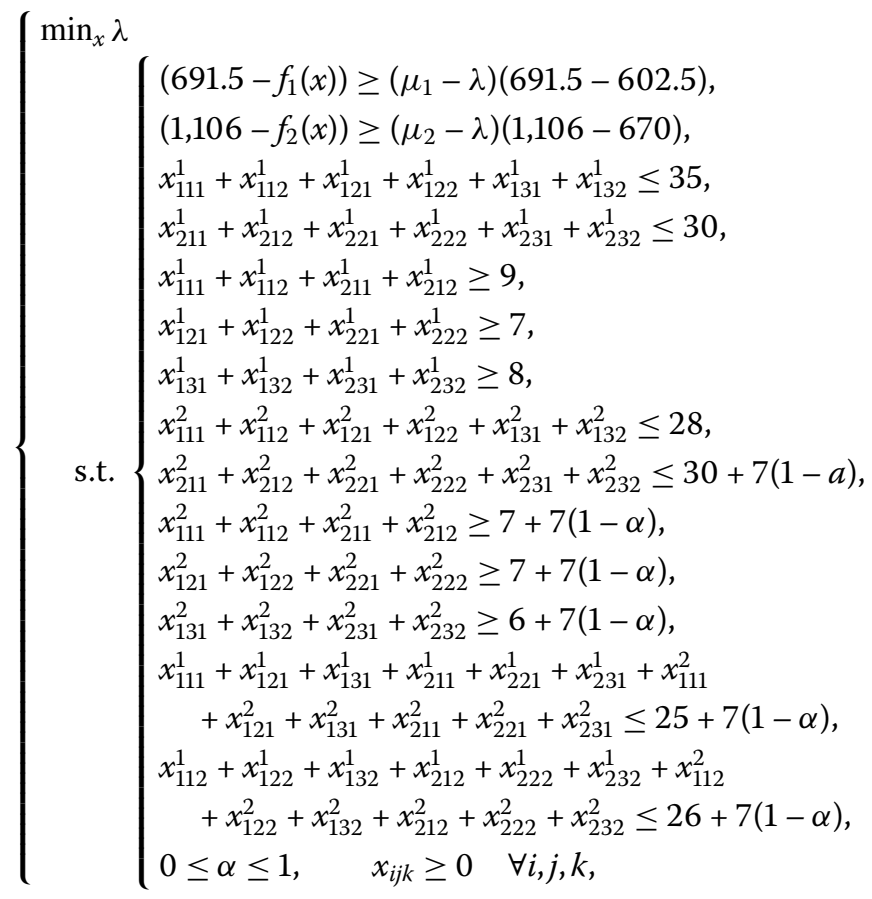

where $\alpha=0.5$ has been calculated in Example 9.1. Here we solve Model-1, to get the satisfied solutions, which are listed in Table 1.

The first line of Table 1 lists each reference value of membership function $\mu_{1}\left(f_{1}\right)$, when the initialized membership function is 1 , the value of objective function $f_{1}(x)$, and its corresponding solution $x$. If the decision maker hopes to improve $f_{2}(x)$ on the basis of sacrifice 
Table 2 Optimum results by using the convex combination method of Model-1

\begin{tabular}{lllll}
\hline $\boldsymbol{w}_{\mathbf{1}}$ & $\boldsymbol{w}_{\mathbf{2}}$ & $\boldsymbol{f}_{\mathbf{1}}$ & $\boldsymbol{f}_{\mathbf{2}}$ & Values of decision variables \\
\hline 0.5 & 0.5 & 691.5 & 670 & $x_{111}^{1}=9, x_{131}^{1}=6.5, x_{222}^{1}=7, x_{232}^{1}=1.5, x_{212}^{2}=10.5, x_{222}^{2}=10.5, x_{231}^{2}=9.5$ \\
0.6 & 0.4 & 688 & 673.5 & $x_{111}^{1}=9, x_{131}^{1}=8, x_{222}^{1}=7, x_{212}^{2}=10.5, x_{221}^{2}=2, x_{222}^{2}=8.5, x_{231}^{2}=9.5$ \\
0.7 & 0.3 & 651 & 748.5 & $x_{111}^{1}=9, x_{131}^{1}=8, x_{222}^{1}=7, x_{212}^{2}=10.5, x_{221}^{2}=10.5, x_{232}^{2}=9.5$ \\
\hline
\end{tabular}

Table 3 Comparison of optimum results of Model-1

\begin{tabular}{llll}
\hline Method & $\boldsymbol{f}_{\mathbf{1}}$ & $\boldsymbol{f}_{\mathbf{2}}$ & Values of decision variables \\
\hline FISM & 630.98 & 799.15 & $x_{111}^{1}=9, x_{121}^{1}=0.91, x_{131}^{1}=8, x_{222}^{1}=6.08$, \\
& & & $x_{121}^{2}=10.5, x_{212}^{2}=10.5, x_{232}^{2}=9.5$ \\
Convex combination & 651 & 748.5 & $\begin{array}{l}x_{111}^{1}=9, x_{131}^{1}=8, x_{222}^{1}=7, x_{212}^{2}=10.5, \\
x_{221}^{2}=10.5, x_{232}^{2}=9.5\end{array}$ \\
Global criteria & 669 & 711.2 & $\begin{array}{l}x_{111}^{1}=9, x_{131}^{1}=6.75, x_{222}^{1}=7, x_{232}^{1}=1.24, \\
\end{array}$ \\
& & & $x_{221}^{2}=7.95, x_{212}^{2}=10, x_{231}^{2}=4.78$, \\
& & $x_{222}^{1}=2.54, x_{232}^{2}=4.71, \mathrm{GC}=0.1265$ \\
\hline
\end{tabular}

$f_{1}(x)$, we may consider resetting the reference value of membership function $\left(\mu_{1}, \mu_{2}\right), e . g$, we set $\left(\mu_{1}, \mu_{2}\right)=(0.9,1)$ or $\left(\mu_{1}, \mu_{2}\right)=(1,0.9)$. The corresponding results are listed in the second and third lines. Suppose that when the reference value of membership function is $\left(\mu_{1}, \mu_{2}\right)=(0.9,1)$, the decision maker is satisfied, then the interactive process is stopped, so we obtain that the satisfied solutions for different items are

$$
\begin{aligned}
& {\left[\begin{array}{lll}
x_{111}^{(1)} & x_{112}^{(1)} & x_{121}^{(1)} \\
x_{122}^{(1)} & x_{131}^{(1)} & x_{132}^{(1)} \\
x_{211}^{(1)} & x_{212}^{(1)} & x_{221}^{(1)} \\
x_{222}^{(1)} & x_{231}^{(1)} & x_{232}^{(1)}
\end{array}\right]=\left[\begin{array}{ccc}
9 & 0 & 0.91 \\
0 & 8 & 0 \\
0 & 0 & 0 \\
6.08 & 0 & 0
\end{array}\right],} \\
& {\left[\begin{array}{lll}
x_{111}^{(2)} & x_{112}^{(2)} & x_{121}^{(2)} \\
x_{122}^{(2)} & x_{131}^{(2)} & x_{132}^{(2)} \\
x_{211}^{(2)} & x_{212}^{(2)} & x_{221}^{(2)} \\
x_{222}^{(2)} & x_{231}^{(2)} & x_{232}^{(2)}
\end{array}\right]=\left[\begin{array}{ccc}
0 & 0 & 10.5 \\
0 & 0 & 0 \\
0 & 10.5 & 0 \\
0 & 0 & 9.5
\end{array}\right]}
\end{aligned}
$$

and the corresponding optimal values for different items are

$$
\left[\begin{array}{ll}
f_{1}^{(1)} & f_{1}^{(2)} \\
f_{2}^{(1)} & f_{2}^{(2)}
\end{array}\right]=\left[\begin{array}{cc}
282.26 & 355.5 \\
352.15 & 447
\end{array}\right] \text {. }
$$

Applying the convex combination method stated in Section 4.3, we get Table 2 for different weights on $f_{1}$ and $f_{2}$. The comparison between the optimum results calculated by different methods for Model-1 is given in Table 3.

\subsection{Input data for Model-2}

Let us consider a multi-objective single-item solid transportation problem with three origins (i.e., $M=3$ ), two destinations (i.e., $N=2$ ) and two types of conveyances (i.e., $K=2$ ). The parameters are given as follows. 
Transportation cost for 1 st objective $\left[c_{i j k}^{(1)}\right]$

$$
\left[\begin{array}{lll}
c_{111}^{(1)} & c_{112}^{(1)} & c_{121}^{(1)} \\
c_{122}^{(1)} & c_{131}^{(1)} & c_{132}^{(1)} \\
c_{211}^{(1)} & c_{212}^{(1)} & c_{221}^{(1)} \\
c_{222}^{(1)} & c_{231}^{(1)} & c_{232}^{(1)}
\end{array}\right]=\left[\begin{array}{ccc}
10 & 12 & 11 \\
13 & 9 & 19 \\
12 & 11 & 13 \\
14 & 13 & 19
\end{array}\right]
$$

Transportation cost for 2 nd objective $\left[c_{i j k}^{(2)}\right]$

$$
\left[\begin{array}{lll}
c_{111}^{(2)} & c_{112}^{(2)} & c_{121}^{(2)} \\
c_{122}^{(2)} & c_{131}^{(2)} & c_{132}^{(2)} \\
c_{211}^{(2)} & c_{212}^{(2)} & c_{221}^{(2)} \\
c_{222}^{(2)} & c_{231}^{(2)} & c_{232}^{(2)}
\end{array}\right]=\left[\begin{array}{lll}
16 & 15 & 12 \\
11 & 14 & 16 \\
15 & 15 & 13 \\
11 & 14 & 13
\end{array}\right]
$$

Amount of items available at origin $\left[a_{i}\right]$

$$
\left[\begin{array}{ll}
a_{1} & a_{2}
\end{array}\right]=\left[\begin{array}{ll}
21 & 20
\end{array}\right] \text {. }
$$

The demand amount of items at destination $\left[b_{j}\right]$

$$
\left[\begin{array}{lll}
b_{1} & b_{2} & b_{3}
\end{array}\right]=\left[\begin{array}{lll}
7 & 5 & 2
\end{array}\right] .
$$

Amount of items transported by conveyances $\left[c_{k}\right]$

$$
\left[\begin{array}{ll}
e_{1} & e_{2}
\end{array}\right]=\left[\begin{array}{ll}
20 & 22
\end{array}\right] \text {. }
$$

Optimum result for Model-2

With the above input data, $f_{1}$ and $f_{2}$ are calculated using the GRG technique, and we get

$$
f_{1}^{U}=345.76, \quad f_{1}^{L}=252.1, \quad f_{2}^{U}=344.20, \quad f_{2}^{L}=323.20 .
$$

So we can get the membership functions of $f_{1}$ and $f_{2}$ (Figure 2 ) as follows:

$$
\mu_{1}\left(f_{1}(x)\right)= \begin{cases}0 & \text { for } f_{1}(x)>345.76 \\ \frac{345.76-f_{1}(x)}{345.76-252.1} & \text { for } 252.1<f_{1}(x)<345.76 \\ 1 & \text { for } f_{1}(x)<252.1\end{cases}
$$
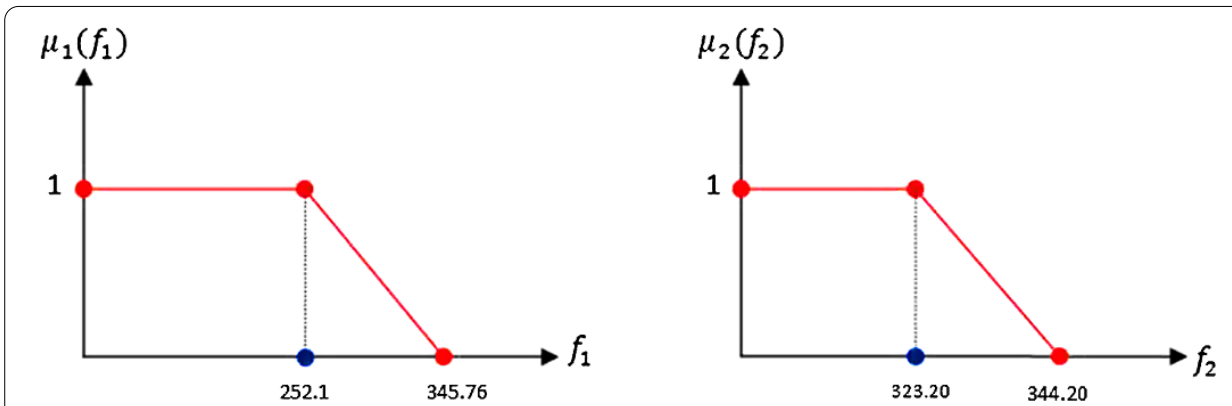

Figure 2 Membership functions of $f_{1}$ and $f_{2}$. 


$$
\mu_{2}\left(f_{2}(x)\right)= \begin{cases}0 & \text { for } f_{2}(x)>344.20 \\ \frac{344.20-f_{2}(x)}{344.20-323.20} & \text { for } 323.20<f_{2}(x)<344.20 \\ 1 & \text { for } f_{2}(x)<323.20\end{cases}
$$

Then we compute the following model to get the interactive satisfied solution:

$$
\left\{\begin{aligned}
\min _{x} \lambda & \left\{\begin{array}{l}
\left(345.76-f_{1}(x)\right) \geq\left(\mu_{1}-\lambda\right)(345.76-252.1), \\
\left(344.20-f_{2}(x)\right) \geq\left(\mu_{2}-\lambda\right)(344.20-323.20), \\
x_{111}+x_{112}+x_{121}+x_{122}+x_{131}+x_{132} \leq 21, \\
x_{211}+x_{212}+x_{221}+x_{222}+x_{231}+x_{232} \leq 20, \\
x_{111}+x_{112}+x_{211}+x_{212} \geq 7+7(1-\alpha), \\
x_{121}+x_{122}+x_{221}+x_{222} \geq 5+7(1-\alpha), \\
x_{131}+x_{132}+x_{231}+x_{232} \geq 2+7(1-\alpha), \\
x_{111}+x_{121}+x_{131}+x_{211}+x_{221}+x_{231} \leq 20+7(1-\alpha), \\
x_{112}+x_{122}+x_{132}+x_{212}+x_{222}+x_{232} \leq 22+7(1-\alpha), \\
x_{i j k} \geq 0 \quad \forall i, j, k, \quad 0 \leq \alpha \leq 1,
\end{array}\right.
\end{aligned}\right.
$$

where $\alpha=0.4974$ has been already calculated in Example 9.2. Here we solve Model-2 to get the satisfied solutions, which are listed in Table 4.

The first line of Table 4 lists each reference value of membership function $\mu_{1}\left(f_{1}\right)$, when the initialized membership function is 1 , the value of objective function $f_{1}(x)$, and its corresponding solution $x$. If the decision maker hopes to improve $f_{2}(x)$ on the basis of sacrifice $f_{1}(x)$, we may consider resetting the reference value of membership function $\left(\mu_{1}, \mu_{2}\right)$, e.g., we set $\left(\mu_{1}, \mu_{2}\right)=(0.9,1)$ or $\left(\mu_{1}, \mu_{2}\right)=(1,0.9)$. The corresponding results are listed in the second and third lines. Suppose that when the reference value of membership function is $\left(\mu_{1}, \mu_{2}\right)=(1,0.90)$, the decision maker is satisfied, then the interactive process is stopped, so we obtain that the satisfied solutions are $x_{121}=1.34, x_{122}=7.16, x_{131}=5.51, x_{212}=10.51$. The corresponding optimal values are

$$
\left[\begin{array}{ll}
f_{1} & f_{2}
\end{array}\right]=\left[\begin{array}{ll}
273.40 & 330.67
\end{array}\right]
$$

Applying the convex combination method stated in Section 4.3, we get Table 5 for different weights on $f_{1}$ and $f_{2}$. The comparison between optimum results calculated by different methods for Model-2 is given in Table 6.

Table 4 Employ the interactive fuzzy satisfied method based on fuzzy inequality constraint for Model-2

\begin{tabular}{llllllll}
\hline $\boldsymbol{\mu}_{\mathbf{1}}$ & $\boldsymbol{\mu}_{\mathbf{2}}$ & $\boldsymbol{f}_{\mathbf{1}}$ & $\boldsymbol{f}_{\mathbf{2}}$ & $\boldsymbol{\mu}_{\mathbf{1}}\left(\boldsymbol{f}_{\mathbf{1}}\right)$ & $\boldsymbol{\mu}_{\mathbf{2}}\left(\boldsymbol{f}_{\mathbf{2}}\right)$ & Values of decision variables & $\boldsymbol{\lambda}$ \\
\hline 1 & 1 & 276.55 & 328.68 & 0.73 & 0.73 & $x_{122}=8.51, x_{131}=5.47, x_{212}=10.51, x_{232}=0.045$ & 0.261 \\
0.9 & 1 & 283.03 & 328.03 & 0.66 & 0.76 & $x_{122}=8.51, x_{131}=4.82, x_{212}=10.51, x_{232}=0.69$ & 0.2302 \\
1 & 0.9 & 273.4 & 330.07 & 0.67 & 0.77 & $x_{121}=1.34, x_{122}=7.16, x_{131}=5.51, x_{212}=10.51$ & 0.227 \\
\hline
\end{tabular}

Table 5 Optimum results by using the convex combination method for Model-2

\begin{tabular}{lllll}
\hline $\boldsymbol{w}_{\mathbf{1}}$ & $\boldsymbol{w}_{\mathbf{2}}$ & $\boldsymbol{f}_{\mathbf{1}}$ & $\boldsymbol{f}_{\mathbf{2}}$ & Values of decision variables \\
\hline 0.5 & 0.5 & 259.06 & 337.24 & $x_{121}=8.51, x_{131}=5.51, x_{212}^{1}=10.51, x_{121}^{1}=8.51$ \\
0.3 & 0.7 & 276.1 & 328.72 & $x_{122}=8.51, x_{131}=5.51, x_{212}^{1}=10.51$ \\
0.6 & 0.4 & 252.1 & 344.20 & $x_{111}=6.96, x_{121}=8.51, x_{131}^{1}=5.51, x_{212}^{1}=3.55$ \\
\hline
\end{tabular}


Table 6 Comparison of optimum results of Model-2

\begin{tabular}{llll}
\hline Method & $\boldsymbol{f}_{\mathbf{1}}$ & $\boldsymbol{f}_{\mathbf{2}}$ & Values of decision variables \\
\hline FISM & 273.40 & 330.07 & $x_{121}=1.34, x_{122}=7.16, x_{131}=5.51, x_{212}=10.51$ \\
Convex combination & 252.1 & 344.20 & $x_{111}=6.96, x_{121}=8.51, x_{131}=5.51, x_{212}=3.55$ \\
Global criteria & 259.06 & 337.24 & $x_{121}=8.51, x_{131}=5.51, x_{212}=10.51, \mathrm{GC}=0.0514$ \\
\hline
\end{tabular}

\subsection{Optimum result for Model-3}

To solve Model-3, we will solve $\operatorname{MSSTP}_{\alpha}$ (Example 9.1) and the optimum solution has come for decision $\alpha^{*}=0.5$, and the optimal solutions for different items are

$$
\begin{aligned}
& {\left[\begin{array}{lll}
x_{111}^{(1)} & x_{112}^{(1)} & x_{121}^{(1)} \\
x_{122}^{(1)} & x_{131}^{(1)} & x_{132}^{(1)} \\
x_{211}^{(1)} & x_{212}^{(1)} & x_{221}^{(1)} \\
x_{222}^{(1)} & x_{231}^{(1)} & x_{232}^{(1)}
\end{array}\right]=\left[\begin{array}{ccc}
0 & 2.70 & 7 \\
0 & 0 & 2.47 \\
6.29 & 0 & 0 \\
0 & 5.52 & 0
\end{array}\right],} \\
& {\left[\begin{array}{lll}
x_{11}^{(2)} & x_{112}^{(2)} & x_{121}^{(2)} \\
x_{122}^{(2)} & x_{131}^{(2)} & x_{132}^{(2)} \\
x_{211}^{(2)} & x_{212}^{(2)} & x_{221}^{(2)} \\
x_{222}^{(2)} & x_{231}^{(2)} & x_{232}^{(2)}
\end{array}\right]=\left[\begin{array}{ccc}
0 & 4.81 & 8.32 \\
2.17 & 0 & 0 \\
0 & 5.68 & 0 \\
0 & 0 & 9.5
\end{array}\right]}
\end{aligned}
$$

and the corresponding optimal values for different items are

$$
\left[\begin{array}{ll}
f_{1}^{(1)} & f_{1}^{(2)} \\
f_{2}^{(1)} & f_{2}^{(2)}
\end{array}\right]=\left[\begin{array}{cc}
247 & 355.5 \\
719.10 & 477.59
\end{array}\right] \text {. }
$$

\section{Conclusion}

The multi-objective multi-item solid transportation problem in fuzzy inequality constraints has been explored in this paper. Three different models have been derived. First, a fuzzy inequality solid transportation problem has been converted to a parametric solid transportation problem using flexible index, and then the fuzzy inequality solid transportation problem has been solved by using the decision making technique. The fuzzy interactive satisfied method, global criterion method and convex combination method have been applied to calculate the optimal compromise solutions of multi-objective STP problem, and then it was solved by using MatLab and Lingo-11.0. The models are illustrated with numerical examples and corresponding results are compared. This paper only researches the problem under fuzzy inequality constraints, and the problem in other more complex environments or multi-objective uncertain transportation problem may become new topics in further research. The present formulation and solution procedures can be applied to other fuzzy transportation models with different fuzzy numbers.

\section{Appendix}

Example 9.1 Consider the following FICP:

$$
\begin{aligned}
& \min 11 x_{111}^{1}+10 x_{112}^{1}+11 x_{121}^{1}+13 x_{122}^{1}+11 x_{131}^{1}+10 x_{132}^{1}+10 x_{211}^{1}+11 x_{212}^{1} \\
& \quad+13 x_{221}^{1}+14 x_{222}^{1}+10 x_{231}^{1}+12 x_{232}^{1}+18 x_{111}^{2}+11 x_{112}^{2}+12 x_{121}^{2}+12 x_{122}^{2} \\
& \quad+13 x_{131}^{2}+15 x_{132}^{2}+12 x_{211}^{2}+11 x_{212}^{2}+13 x_{221}^{2}+14 x_{222}^{2}+15 x_{231}^{2}+12 x_{232}^{2}
\end{aligned}
$$




$$
\begin{aligned}
\text { s.t. } & x_{111}^{1}+x_{112}^{1}+x_{121}^{1}+x_{122}^{1}+x_{131}^{1}+x_{132}^{1} \lesssim 35, \\
& x_{211}^{1}+x_{212}^{1}+x_{221}^{1}+x_{222}^{1}+x_{231}^{1}+x_{232}^{1} \lesssim 30, \\
& x_{111}^{1}+x_{112}^{1}+x_{211}^{1}+x_{212}^{1} \gtrsim 9, \\
& x_{121}^{1}+x_{122}^{1}+x_{221}^{1}+x_{222}^{1} \gtrsim 7, \\
& x_{131}^{1}+x_{132}^{1}+x_{231}^{1}+x_{232}^{1} \gtrsim 8, \\
& x_{111}^{2}+x_{112}^{2}+x_{121}^{2}+x_{122}^{2}+x_{131}^{2}+x_{132}^{2} \lesssim 28, \\
& x_{211}^{2}+x_{212}^{2}+x_{221}^{2}+x_{222}^{2}+x_{231}^{2}+x_{232}^{2} \lesssim 30, \\
& x_{111}^{2}+x_{112}^{2}+x_{211}^{2}+x_{212}^{2} \gtrsim 7, \\
& x_{121}^{2}+x_{122}^{2}+x_{221}^{2}+x_{222}^{2} \gtrsim 7, \\
& x_{131}^{2}+x_{132}^{2}+x_{231}^{2}+x_{232}^{2} \gtrsim 6, \\
& x_{111}^{1}+x_{121}^{1}+x_{131}^{1}+x_{211}^{1}+x_{221}^{1}+x_{231}^{1}+x_{111}^{2} \\
& +x_{121}^{2}+x_{131}^{2}+x_{211}^{2}+x_{221}^{2}+x_{231}^{2} \lesssim 25, \\
& x_{112}^{1}+x_{122}^{1}+x_{132}^{1}+x_{212}^{1}+x_{222}^{1}+x_{232}^{1}+x_{112}^{2} \\
& +x_{122}^{2}+x_{132}^{2}+x_{212}^{2}+x_{222}^{2}+x_{232}^{2} \lesssim 26, \\
& x_{i j k}^{p} \geq 0 \quad \forall i, j, k \text { and } p=1,2, \quad d=(0,0,0,0,0,0,7,7,7,7,7,7) .
\end{aligned}
$$

The corresponding parametric linear programming problem of $\widetilde{M M S T P}$ is presented as follows:

$$
\begin{aligned}
\min 11 & x_{111}^{1}+10 x_{112}^{1}+11 x_{121}^{1}+13 x_{122}^{1}+11 x_{131}^{1}+10 x_{132}^{1} \\
& +10 x_{211}^{1}+11 x_{212}^{1}+13 x_{221}^{1}+14 x_{222}^{1}+10 x_{231}^{1}+12 x_{232}^{1} \\
& +18 x_{111}^{2}+11 x_{112}^{2}+12 x_{121}^{2}+12 x_{122}^{2}+13 x_{131}^{2}+15 x_{132}^{2} \\
& +12 x_{211}^{2}+11 x_{212}^{2}+13 x_{221}^{2}+14 x_{222}^{2}+15 x_{231}^{2}+12 x_{232}^{2} \\
\text { s.t. } & x_{111}^{1}+x_{112}^{1}+x_{121}^{1}+x_{122}^{1}+x_{131}^{1}+x_{132}^{1} \leq 35, \\
& x_{211}^{1}+x_{212}^{1}+x_{221}^{1}+x_{222}^{1}+x_{231}^{1}+x_{232}^{1} \leq 30, \\
& x_{111}^{1}+x_{112}^{1}+x_{211}^{1}+x_{212}^{1} \geq 9, \\
& x_{121}^{1}+x_{122}^{1}+x_{221}^{1}+x_{222}^{1} \geq 7, \\
& x_{131}^{1}+x_{132}^{1}+x_{231}^{1}+x_{232}^{1} \geq 8, \\
& x_{111}^{2}+x_{112}^{2}+x_{121}^{2}+x_{122}^{2}+x_{131}^{2}+x_{132}^{2} \leq 28, \\
& x_{211}^{2}+x_{212}^{2}+x_{221}^{2}+x_{222}^{2}+x_{231}^{2}+x_{232}^{2} \leq 30+7(1-a), \\
& x_{111}^{2}+x_{112}^{2}+x_{211}^{2}+x_{212}^{2} \geq 7+7(1-\alpha), \\
& x_{121}^{2}+x_{122}^{2}+x_{221}^{2}+x_{222}^{2} \geq 7+7(1-\alpha), \\
& x_{131}^{2}+x_{132}^{2}+x_{231}^{2}+x_{232}^{2} \geq 6+7(1-\alpha), \\
& x_{111}^{1}+x_{121}^{1}+x_{131}^{1}+x_{211}^{1}+x_{221}^{1}+x_{231}^{1}+x_{111}^{2} \\
\text { MMSTP } \alpha &
\end{aligned}
$$




$$
\begin{aligned}
& +x_{121}^{2}+x_{131}^{2}+x_{211}^{2}+x_{221}^{2}+x_{231}^{2} \leq 25+7(1-\alpha), \\
& x_{112}^{1}+x_{122}^{1}+x_{132}^{1}+x_{212}^{1}+x_{222}^{1}+x_{232}^{1}+x_{112}^{2} \\
& +x_{122}^{2}+x_{132}^{2}+x_{212}^{2}+x_{222}^{2}+x_{232}^{2} \leq 26+7(1-\alpha), \\
& x_{i j k}^{p} \geq 0 \quad \forall i, j, k, p=1,2 \quad \text { and } \quad 0 \leq \alpha \leq 1 .
\end{aligned}
$$

Now, using the algorithm given in Section 2.1, we obtain $Z_{0}=725, Z_{1}=480$ and $d_{0}=245$ by calculating $\left(M M S T P_{0}\right)$ and $\left(M M S T P_{1}\right)$ corresponding to $(25)$. Let the inverse of the optimal matrix in $\left(M M S T P_{0}\right)$ be

$$
B_{0}^{-1}=\left(\begin{array}{cccccccccccc}
1 & 0 & 0 & 1 & 0 & 0 & 0 & -1 & 0 & -1 & 0 & -1 \\
0 & 1 & 1 & 0 & 1 & 0 & 0 & 1 & 0 & 1 & 0 & 1 \\
0 & 0 & 0 & 0 & -1 & 0 & 0 & -1 & 0 & -1 & 0 & -1 \\
0 & 0 & 0 & -1 & 0 & 0 & 0 & 0 & 0 & 0 & 0 & 0 \\
0 & 0 & 0 & 0 & 0 & 0 & 0 & 1 & 0 & 1 & 0 & 1 \\
0 & 0 & 0 & 0 & 0 & 1 & 0 & 1 & 1 & 0 & 0 & 0 \\
0 & 0 & 0 & 0 & 0 & 0 & 1 & 0 & 0 & 1 & 0 & 0 \\
0 & 0 & 0 & 0 & 0 & 0 & 0 & -1 & 0 & 0 & 0 & 0 \\
0 & 0 & 0 & 0 & 0 & 0 & 0 & 0 & -1 & 0 & 0 & 0 \\
0 & 0 & 0 & 0 & 0 & 0 & 0 & 0 & 0 & -1 & 0 & 0 \\
0 & 0 & 1 & 1 & 1 & 0 & 0 & 1 & 1 & 1 & 1 & 1 \\
0 & 0 & -1 & 0 & 0 & 0 & 0 & 0 & 0 & 0 & 0 & 0
\end{array}\right) .
$$

Now, calculating the equations $\left[B_{0}^{-1}(35,30,9,7,8,28,30+7(1-\alpha), 7+7(1-\alpha), 7+7(1-\right.$ $\alpha), 6+7(1-\alpha), 25+7(1-\alpha), 26+7(1-\alpha))]_{i}=0(i=1,2, \ldots, 12)$, respectively, we obtain $\alpha_{1}=0.8571$ assume $\alpha_{0}=0$ and $\alpha_{2}=1$.

Now, solving $\left(M M S T P_{\alpha_{1}}\right)$, we get an optimal solution and an optimal value as $Z_{\alpha_{1}}=$ $Z_{0.8571}=515.01$.

Now, $Z_{0.8571}=515.01<Z_{1}+0.8571 \times 245=689.98$, so we stop here and calculate optimal decision $\alpha^{*}$. Now

$$
\begin{aligned}
\alpha^{*} & =\frac{Z_{1} \alpha_{1}-Z_{1} \alpha_{0}-Z_{\alpha_{0}} \alpha_{1}+Z_{\alpha_{1}} \alpha_{0}}{Z_{\alpha_{1}}-Z_{\alpha_{0}}-\alpha_{1} d_{0}+\alpha_{0} d_{0}} \\
& =0.5
\end{aligned}
$$

Now, solving $\left(M M S T P_{0.5}\right)$, we obtain the optimal value $Z_{0.5}^{*}=602.5$.

Example 9.2 Consider the following FICP:

$$
\begin{aligned}
& \min 10 x_{111}+12 x_{112}+11 x_{121}+13 x_{122} \\
& +9 x_{131}+19 x_{132}+12 x_{211}+11 x_{212} \\
& +13 x_{221}+14 x_{222}+13 x_{231}+19 x_{232} \\
& \text { s.t. } x_{111}+x_{112}+x_{121}+x_{122}+x_{131}+x_{132} \lesssim 21, \\
& x_{211}+x_{212}+x_{221}+x_{222}+x_{231}+x_{232} \lesssim 20
\end{aligned}
$$




$$
\begin{array}{ll} 
& x_{111}+x_{112}+x_{211}+x_{212} \gtrsim 7 \\
& x_{121}+x_{122}+x_{221}+x_{222} \gtrsim 5, \\
& x_{131}+x_{132}+x_{231}+x_{232} \gtrsim 2 \\
& x_{111}+x_{121}+x 131+x_{211}+x_{221}+x_{231} \lesssim 20, \\
& x_{112}+x_{122}+x_{132}+x_{212}+x_{222}+x_{232} \lesssim 22, \\
& x_{i j k} \geq 0 \quad \forall i, j, k, \quad d=(0,0,7,7,7,7,7) .
\end{array}
$$

The corresponding parametric linear programming problem of $\widehat{M S S T P}$ is presented as follows:

$$
\begin{aligned}
\min 10 & x_{111}+12 x_{112}+11 x_{121}+13 x_{122} \\
& +9 x_{131}+19 x_{132}+12 x_{211}+11 x_{212} \\
& +13 x_{221}+14 x_{222}+13 x_{231}+19 x_{232} \\
\text { s.t. } & x_{111}+x_{112}+x_{121}+x_{122}+x_{131}+x_{132} \leq 21, \\
& x_{211}+x_{212}+x_{221}+x_{222}+x_{231}+x_{232} \leq 20, \\
& x_{111}+x_{112}+x_{211}+x_{212} \geq 7+7(1-\alpha), \\
& x_{121}+x_{122}+x_{221}+x_{222} 5+7(1-\alpha), \\
& x_{131}+x_{132}+x_{231}+x_{232} \geq 2+7(1-\alpha), \\
& x_{111}+x_{121}+x 131+x_{211}+x_{221}+x_{231} \leq 20+7(1-\alpha), \\
& x_{112}+x_{122}+x_{132}+x_{212}+x_{222}+x_{232} \leq 22+7(1-\alpha), \\
& x_{i j k} \geq 0 \quad \forall i, j, k, \quad 0 \leq \alpha \leq 1 .
\end{aligned}
$$

Now, using the algorithm given in Section 2.1, we obtain $Z_{0}=367, Z_{1}=143$ and $d_{0}=224$ by calculating $\left(M S S T P_{0}\right)$ and $\left(M S S T P_{1}\right)$ corresponding to $(27)$. The inverse of the optimal matrix in $\left(M_{S S T P}\right)$ is

$$
B_{0}^{-1}=\left(\begin{array}{ccccccc}
-1 & 0 & -1 & -1 & -1 & 0 & 0 \\
1 & 1 & 1 & 1 & 1 & 0 & 0 \\
1 & 0 & 0 & 1 & 1 & 0 & 0 \\
0 & 0 & 0 & -1 & 0 & 0 & 0 \\
0 & 0 & 0 & 0 & -1 & 0 & 0 \\
-1 & 0 & 0 & 0 & 0 & 1 & 0 \\
1 & 0 & 1 & 1 & 1 & 0 & 1
\end{array}\right)
$$

Now, calculating the equations $\left[B_{0}^{-1}(21,20,7+7(1-\alpha), 5+7(1-\alpha), 2+7(1-\alpha), 20+7(1-\right.$ $\alpha), 22+7(1-\alpha))]_{i}=0(i=1,2, \ldots, 7)$, respectively, we obtain $\alpha_{1}=0.8571$ assume $\alpha_{0}=0$ and $\alpha_{2}=1$.

Now, solving $\left(M S S T P_{\alpha_{1}}\right)$, we get an optimal solution as $x_{122}=9, x_{311}=9$ and $x_{321}=2$ and an optimal value as $Z_{\alpha_{1}}=Z_{0.8571}=173.009$. 
Now $Z_{0.8571}=173.009<Z_{1}+0.8571 \times 224=334.99$, so we stop here and calculate optimal decision $\alpha^{*}$. Now

$$
\begin{aligned}
\alpha^{*} & =\frac{Z_{1} \alpha_{1}-Z_{1} \alpha_{0}-Z_{\alpha_{0}} \alpha_{1}+Z_{\alpha_{1}} \alpha_{0}}{Z_{\alpha_{1}}-Z_{\alpha_{0}}-\alpha_{1} d_{0}+\alpha_{0} d_{0}} \\
& =0.4974 .
\end{aligned}
$$

Now, solving $\left(\operatorname{MSSTP}_{0.4974}\right)$, we obtain an optimal value as $Z_{0.4974}^{*}=252.1$.

\section{Competing interests}

The authors declare that they have no competing interests.

\section{Authors' contributions}

All authors contributed equally to the writing of this paper. All authors read and approved the final manuscript.

\section{Author details}

${ }^{1}$ Department of Mathematics, Heritage Institute of Technology, Anandapur, Kolkata, West Bengal 700107, India.

${ }^{2}$ Department of Applied Science, Haldia Institute of Technology, Purba Midnapur, Haldia, West Bengal 721657, India.

${ }^{3}$ Department of Mathematics, Bengal Engineering and Science University, Shibpur, Howrah, West Bengal 711103, India.

\section{Acknowledgements}

The authors sincerely thank the anonymous reviewers and editor-in-chief for their careful reading, constructive comments and fruitful suggestions. The first two authors are also thankful to Ms. Priyanka Dey, Assistant Professor, Haldia Institute of Technology for advices on grammatical errors and organization of the paper.

\section{Received: 23 April 2014 Accepted: 19 August 2014 Published: 03 Sep 2014}

\section{References}

1. Haley, K: The solid transportation problem. Oper. Res. 10, 448-463 (1962)

2. Ojha, A, Das, B, Mondal, S, Maiti, M: A solid transportation problem for an item with fixed charge, vehicle cost and price discounted varying charge using genetic algorithm. Appl. Soft Comput. 10, 100-110 (2010)

3. Pramanik, S, Jana, DK, Maiti, K: A multi objective solid transportation problem in fuzzy, bi-fuzzy environment via genetic algorithm. Int. J. Adv. Oper. Manag. 6(1), 4-26 (2014)

4. Bit, AK, Biswal, MP, Alam, SS: Fuzzy programming approach to multi-objective solid transportation problem. Fuzzy Sets Syst. 57, 183-194 (1993)

5. Ida, K, Gen, M, Li, Y: Neural networks for solving multicriteria solid transportation problem. Comput. Ind. Eng. 31, 873-877 (1996)

6. Gao, SP, Liu, SY: Two-phase fuzzy algorithms for multi-objective transportation problem. J. Fuzzy Math. 12, 147-155 (2004)

7. Tao, Z, Xu, J: A class of rough multiple objective programming and its application to solid transportation problem. Inf. Sci. 188, 215-235 (2012)

8. Kundu, P, Kar, S, Maiti, M: Multi-objective multi-item solid transportation problem in fuzzy environment. Appl. Math. Model. 37, 2028-2038 (2013)

9. Pramanik, S, Jana, DK, Maiti, M: Multi-objective solid transportation problem in imprecise environment. J. Transp. Secur. 6, 131-150 (2013)

10. Sancak, E, Salman, S: Multi-item dynamic lot-sizing with delayed transportation policy. Int. J. Prod. Econ. 131, 595-603 (2011)

11. Zimmermann, HJ: Fuzzy programming and linear programming with several objective functions. Fuzzy Sets Syst. 1, 45-55 (1978)

12. Zimmermann, HJ: Description and optimization of fuzzy systems. Int. J. Gen. Syst. 2, 209-215 (1976)

13. Cao, BY: Optimal Models and Methods with Fuzzy Quantities. Springer, Berlin (2010)

14. Sakawa, K: Fuzzy Sets and Interactive Multiobjective Optimization. Plenum, New York (1993)

15. Xu, J, Zhou, X: Fuzzy Like Multiple Objective Decision Making. Springer, Berlin (2011) 